\title{
TOPICAL MANAGEMENT OF RECURRENT APHTHOUS STOMATITIS
}

\author{
Khalid Al-Johani*
}

\begin{abstract}
Recurrent aphthous stomatitis (RAS) is a common condition that is affecting young adults. The management of RAS aims at identifying and controlling the possible predisposing factors, exclude possible underlying systemic causes, control pain and to accelerate healing.

A wide variety of systemic and topical agents has been suggested for the treatment of RAS. Although there are several trials employing different systemic and/or topical agents in the treatment of RAS, there is no strong evidence on the superiority of any therapeutic or curative agent for this disorder.

Topical agents may be sufficient to lessen the pain in some patients; however systemic corticosteroids/immunosuppressant agents may be necessary to control pain in others. Therefore, the severity and frequency of ulcers may be used as guidance in the management of RAS patients. This paper reviews the available topical agents that have been used in the management of RAS.
\end{abstract}

\section{INTRODUCTION}

The management of recurrent aphthous stomatitis (RAS) aims to identify and control the possible predisposing factors, exclude possible underlying systemic causes, control pain, accelerate ulcer healing and prevent secondary infection (Jurge et al., 2006; Belenguer-Guallar et al., 2014). RAS lesions are self-limiting and tend to recur and heal without scarring. However, major lesions may heal with scar in some instances, and with time, a group of patients may adapt to compromised mildmoderate pain and necessitate no treatment.
A wide variety of systemic and topical drugs/ herbal agents have been suggested for the treatment of RAS. Although there are numerous randomizedcontrolled trials (RCTs) employing different systemic and/or topical agents in the treatment of RAS, there is no strong evidence on the superiority of any therapeutic or curative agent for this disorder. Although there is a consensus on the diagnosis and management of RAS (Scully et al., 2003), in practice, there is wide variability in the management between different specialists and clinicians. There is great variability in the severity, frequency and duration of mouth ulcers. In addition, there are

* Assistant Professor, Department of Oral Diagnostic Sciences, Faculty of Dentistry- King Abdulaziz University 
differences in response between RAS patients. Hence, where topical agents (antiseptic, analgesic or topical corticosteroids) may be sufficient to lessen the pain in some patients, systemic corticosteroids/ immunosuppressant agents may be necessary to control pain in others. Therefore, the severity and frequency of ulcers may be used as guidance in the management of RAS patients.

A review based on evidence levels 1 and 2 (metaanalyses, systematic reviews, phase I and II randomized clinical trials, cohort studies and casecontrol studies) suggests that therapy with either topical or systemic agents has to employ only in cases with continuous attacks. Treatment always initiated with topical agents and to reserve systemic therapies with patients with constant and aggressive outbreaks (Belenguer-Guallar et al., 2014).

\section{Spectrum of topical agents in the management of RAS}

\section{Topical corticosteroids}

Topical corticosteroids are considered the mainstay of treatment and have been reported to lessen pain and accelerate healing. A consensus approach suggests that topical corticosteroids with or without other immunomodulatory topical agents may control RAS symptoms in the majority of patients (Scully et al., 2003).

Different topical corticosteroid formulations and agents have been used in the management of RAS, including hydrocortisone mouthwash (Holbrook et al., 1998), clobetasol (Lozada-Nur et al., 1991; Lo Muzio et al., 2001), fluocinonide (Pimlott and Walker, 1983), mometasone furoate (Teixeira et al., 1999), topical dexamethasone and triamcinolone acetonide (Yel et al., 1994; Al-Na'mah et al., 2009; Keenan, 2012).

For example, 40 patients who had recurrent oral ulcers for a mean period of 11.1 years were prescribed topical triamcinolone acetonide $(0.1 \%$ or $0.2 \%$ aqueous suspension) alone or in combination with prednisone $(40-60 \mathrm{mg})$. At the end of the follow-up period (mean of 22.9 months), most patients $(34 / 40 ; 85 \%)$ had complete healing of their ulcers presented at initial consultation with fewer new ulcers, while 5 patients had partial response to this topical therapy. Candidiasis was evident in five patients as a complication of topical corticosteroids (Vincent and Lilly, 1992).

In a RCT, topical clobetasol propionate (3 different preparations, 2-3 times/day) was effective in the management of 30 patients. Clobetasol in an adhesive denture paste, in comparison to other preparations, induced quick pain relief (Lo Muzio et al., 2001). Acute pseudomembranous candidiasis was evident in $18.5 \%$ of the patients in this study, which was effectively managed by miconazole and chlorhexidine (Lo Muzio et al., 2001). In another study, clobetasol propionate $(0.05 \%)$ was found to be as effective as amlexanox (5\%) in lessening the painful symptoms of RAS as well as healing of ulcers (Rodríguez et al., 2007). In a study by Abbasi et al. adcortyl (Triamcinalone) was effective as amlexanox in relieving pain and reducing the ulcer size (Abbasi et al., 2016).

In a study in which dexamethasone ointment was assessed among 810 RAS patients in a randomized, double-blinded, multicenter clinical trial to compare the efficacy and safety of dexamethasone ointment with placebo (3 times a day for 5 days), dexamethasone ointment was found to be efficient and safe with no severe adverse effect and with no serum dexamethasone detection (Liu et al., 2012). In another study, dexamethasone ointment was efficient and safe in reducing ulcer size and lessening symptoms in RAS patients (Keenan, 2012).

A systematic review showed that studies concerning the effect of topical corticosteroids in the management of RAS are inconsistent and not conclusive (Quijano and Rodríguez, 2008). However, these agents result in reduced time of ulcer healing in RAS patients (Quijano and Rodríguez, 2008). 


\section{Antimicrobial}

Topical antiseptic agents (e.g., chlorhexidine and triclosan) can be used for symptomatic relief of RAS pain. Several open and RCTs investigated the effect of chlorhexidine in patients with RAS (Addy and Hunter, 1987; Edres et al., 1997). It has been reported to lessen ulcer pain (Edres et al., 1997). In addition, chlorhexidine mouthwash was as effective as triamcinolone acetonide $(0.025 \%)$ in a cohort of RAS patients (Miles et al., 1993). Triclosan, in a double-blind cross-over clinical trial, employing 30 subjects, resulted in a reduction in ulcer number (Skaare et al., 1996).

Tetracycline, Minocycline, Doxycycline

Tetracyclines inhibit matrix metalloproteinases (degradative enzymes of the extracellular matrix), which play a role in tissue inflammation and contribute to ulcer formation (Vidal et al., 2007; Skulason et al., 2009).

In a double-blind trial, tetracycline, as a suspension, lessened the symptoms of a cohort of RAS patients; however, as with most other agents, it failed to stop ulcer recurrences (Graykowski and Kingman, 1978). However, tetracycline mouthwash $(0.25 \%)$ was less effective than minocycline mouthwash $(0.2 \%)$ in the control of pain and duration of ulcers in 17 patients in a randomized cross-over study (Gorsky et al., 2007).

Topical minocycline $(0.2 \%)$ was reportedly more effective than placebo mouthwash in reducing the pain severity and ulcer duration in a randomized trial of 33 patients (Gorsky et al., 2008). However, more recently, it was found that $0.5 \%$ minocycline mouthwash is more effective than $0.2 \%$ minocycline (Yarom et al., 2017).

It has also been reported that a single application of doxycycline hyclate decreases pain and improves the healing of RAS lesions (Vijayabala et al., 2013). Skulason and co-workers (2009) conducted a randomized, double-blind, placebo-controlled trial with low-dose doxycycline in an adhesive gel that resulted in healing of mucosal ulcers in $68 \%$ of patients within 3 days of commencing treatment, while just quarter of the patients on placebo had resolution of oral ulceration.

\section{Amlexanox}

Amlexanox is an anti-allergic and antiinflammatory topical agent that has been suggested for the management of RAS (Greer et al., 1993; Khandwala et al., 1997; Bell, 2005; Murray et al., 2005; Murray et al., 2006; Liu et al., 2006; Rodríguez et al., 2007; Meng et al., 2009). Amlexanox, at the onset of the prodromal stage, reportedly prevents the development of ulcers and significantly reduces symptoms if ulcers develop (Murray et al., 2005). Greer and colleagues (1993) in a double-blind placebo controlled clinical trial concluded that amlexanox is an effective and tolerable agent in the treatment of RAS patients.

In a large multicenter randomized, double-blind clinical trial employing 1335 RAS sufferers, 5\% amlexanox paste was found to accelerate the healing and resolution of pain of ulcers within 48 hours of development (Khandwala et al., 1997).

Murray and colleagues (2006), in a randomized controlled trial, used amlexanox (4 times/day for 3 days) in the management of RAS. They employed thermographic imaging to identify ulcers in the prodromal stage in 52 patients. The number of patients who developed an ulcer by the fourth day in the amlexanox group was lower than those who received only vehicle treatment (50\% and 69\%, respectively). In another large randomized, double-blind, vehicle-controlled trial (Liu et al., 2006), 104 patients used amlexanox oral adhesive tablets (4 times a day for 5 days) and 108 used vehicle. Liu and coworkers (2006) found amlexanox to be effective and safe in lessening patients' symptoms and signs.

Additionally, in a multicenter, double-blind randomized trial of 95 RAS patients, 5\% amlexanox (5\%) was found to be effective and as potent as topical corticosteroids $(0.05 \%$ clobetasol propionate $)$ in 
reducing pain and ulcer size (Rodríguez et al., 2007). In a randomized, blinded, placebo-controlled, multicenter trial with 216 subjects, amlexanox pellicles (4 times a day for 5 days) were found to be effective and safe in reducing pain and ulcer size (Meng et al., 2009). In another randomized, doubleblind, vehicle-controlled, unparallel multicenter clinical trial, amlexanox oral adhesive tablets were found to be effective and safe in the management of RAS (Liu et al., 2006).

In a few other studies, 5\% amlexanox oral paste was clinically effective in the management of RAS (Bhat and Sujatha., 2013; Darshan et al., 2014) and was reported to reduce the frequency, duration and symptoms with no adverse side effects (Greer et al., 1993; Darshan et al., 2014). Likewise, in another study, amlexanox, as an oral adhesive pellicle, was found to be as effective and safe as amlexanox oral adhesive tablets and more comfortable (Meng et al., 2009).

In a study by Rodríguez et al., 5\% amlexanox or a $0.05 \%$ clobetasol propionate both relieved pain and reduced the ulcer size, with no significant differences between patients employing them (Rodríguez et al., 2007). In studies conducted by Murray et al, OraDisc (2 mg amlexanox) (Murray et al., 2006) and Aphtheal (5\% amlexanox paste) (Murray et al., 2005) found that preventing the progression of RAS in the prodromal phase into the ulcer phase in comparison with no treatment or vehicle alone and amlexanox significantly reduced symptoms if ulcers develop (Murray et al., 2006).

\section{Hyaluronic acid}

In a large randomized, controlled, doubleblind trial, topical hyaluronic acid was used in the management of 120 RAS patients. Hyaluronic acid resulted in the reduction of ulcers more than observed with those on placebo (Nolan et al., 2006). However, hyaluronic acid and placebo resulted in a significant lessening of pain after commencing treatment (Nolan et al., 2006). In a cohort of 33 RAS or Behçet's disease patients, topical hyaluronic acid
(0.2\%; twice/daily for 14 days) resulted in lessening the symptoms in most patients (75.8\%), as reported with a VAS, as well as a reduction in the number of ulcers and the healing period. Specifically, the authors reported a reduction in ulcer numbers in $57.6 \%$ of the patients, and most ulcers (78.8\%) showed a decrease in size, and the treatment was without any adverse side effects (Lee et al., 2008).

In a systematic review on the effects of hyaluronic acid on painful oral lesions, including recurrent aphthous stomatitis, both subjective and objective parameters were significantly improved (Casale et al., 2017).

\section{Chemical cautery}

Coagulation, employing chemical cautery, has been suggested to be effective in the management of RAS.

\section{Debacterol}

Debacterol (a liquid, topical, debriding agent composed of sulfonated phenolics-50\% and sulfuric acid-30\%) was found to be more effective than triamcinolone acetonide in lessening RAS pain in a cohort of 60 patients (Rhodus and Bereuter, 1998). More than $70 \%$ and less than $20 \%$ of patients had lessening of their symptoms by day 3 of commencing debacterol and triamcinolone acetonide, respectively. However, there was no difference in ulcer size between the two therapeutic agents (Rhodus and Bereuter, 1998).

\section{Silver nitrate cautery}

Silver nitrate cautery may be an effective option for symptomatic pain relief in RAS patients (Alidaee et al., 2005; Soylu Özler, 2014),

In a randomized, single-blinded controlled study, silver nitrate cautery resulted in $70 \%$ lessening of oral pain after 24 hours of the treatment procedure (Alidaee et al., 2005), while just $11 \%$ of patients receiving placebo reported a reduction in symptoms. However, within a week, there was no 
difference between the two groups regarding reepithelialization. The authors concluded that silver nitrate reduced ulcer pain but did not shorten the healing time. Additionally, the authors note that it is a rapid, simple and cost-effective means of treatment in patients with sporadic RAS (Alidaee et al., 2005).

Silver nitrate cautery produced a more statistically significant reduction in pain in comparison with people who were treated with placebo sticks on the first to the seventh day. RAS lesions were completely reepithelialized on the seventh day in $60 \%$ of patients managed by Silver nitrate and in $32 \%$ of patients in the placebo group (mean healing time of ulcers, reported by the Silver nitrate group, was 2.7 days (range 2-4) and in the placebo group, 5.5 days (range 4-7)) with no adverse side effects in both groups (Soylu Özler, 2014).

\section{Laser therapy}

Many studies have evaluated the use of different types and regimens of laser therapy in the management of patients with RAS (Tezel et al., 2009; Prasad and Pai, 2013; Albrektson et al., 2014, Nasry et al., 2016, Pentapati et al., 2016, Zeini Jahromi et al., 2017, Suter et al., 2017). A single application with a non-thermal, non-ablative $\mathrm{CO} 2$ laser therapy may accelerate healing without any adverse effect (Zand et al., 2012). CO2 laser therapy has been reported to give immediate pain relief (Prasad and Pai, 2013).

Low-level laser therapy can accelerate healing, decrease the pain, size, and recurrence of RAS lesions (Anand et al, 2013; Albrektson et al., 2014). low-level laser therapy (wavelength, $809 \mathrm{~nm}$; power, $60 \mathrm{~mW}$; frequency, $1800 \mathrm{~Hz}$; duration, 80 seconds; dose, $6.3 \mathrm{~J} / \mathrm{cm}(2))$ in a randomized controlled trial found to be effective in reducing the pain and the inconvenience of eating, drinking, and brushing for patients with RAS (Albrektson et al., 2014).

In a study where laser therapy is used as an alternative method in the treatment of RAS.
Er,Cr:YSGG (0.25 W without water) and a $940 \mathrm{~nm}$ diode laser may be appropriate to reduce pain, burn sensation and accelerate the healing of RAS (Misra et al., 2013; Yilmaz et al., 2017).

In a randomized clinical trial, diode laser treatment was found to be better with a statistically significant reduction in pain and ulcer size than treatment with adhesive pastes of A. nilotica and Glycyrrhiza glabra, adhesive oral tablets of $2 \mathrm{mg}$ Amlexanox or patients just received a placebo adhesive tablet (Nasry et al., 2016).

Low-level laser therapy was as effective as amlexanox in relieving RAS pain, and both were effective, subjectively and objectively in the management of RAS (Jijin et al., 2016).

In a systematic review on the effect of laser on recurrent aphthous stomatitis (CO2 laser, Nd:YAG laser and diode laser), laser was found to be effective in the management of oral lesions (Suter et al., 2017), and in another systematic review on the low-level lasers, Najeeb and coworker (2016) concluded that various types of lasers are providing immediate pain relief and that carbon dioxide $(\mathrm{CO} 2)$ lasers require a short application time.

The laser therapy has superiority in relieving ulcer pain and shortening healing time when compared with other treatment modalities (e.g., $0.1 \%$ triamcinolone acetonide, Granofurin and solcoseryl, amlexanox oral paste, inactive laser therapy, etc.). Although laser therapy is a promising effective treatment for RAS, high-quality clinical studies with large sample sizes must be performed to confirm the effectiveness of this therapy (Han et al., 2016).

Because there is a large variation in the different regimens in various publications in this field, it has been recommended to standardize the laser type, wavelength, power output and applied energy in the management of RAS to improve patient management (Suter et al., 2017). 


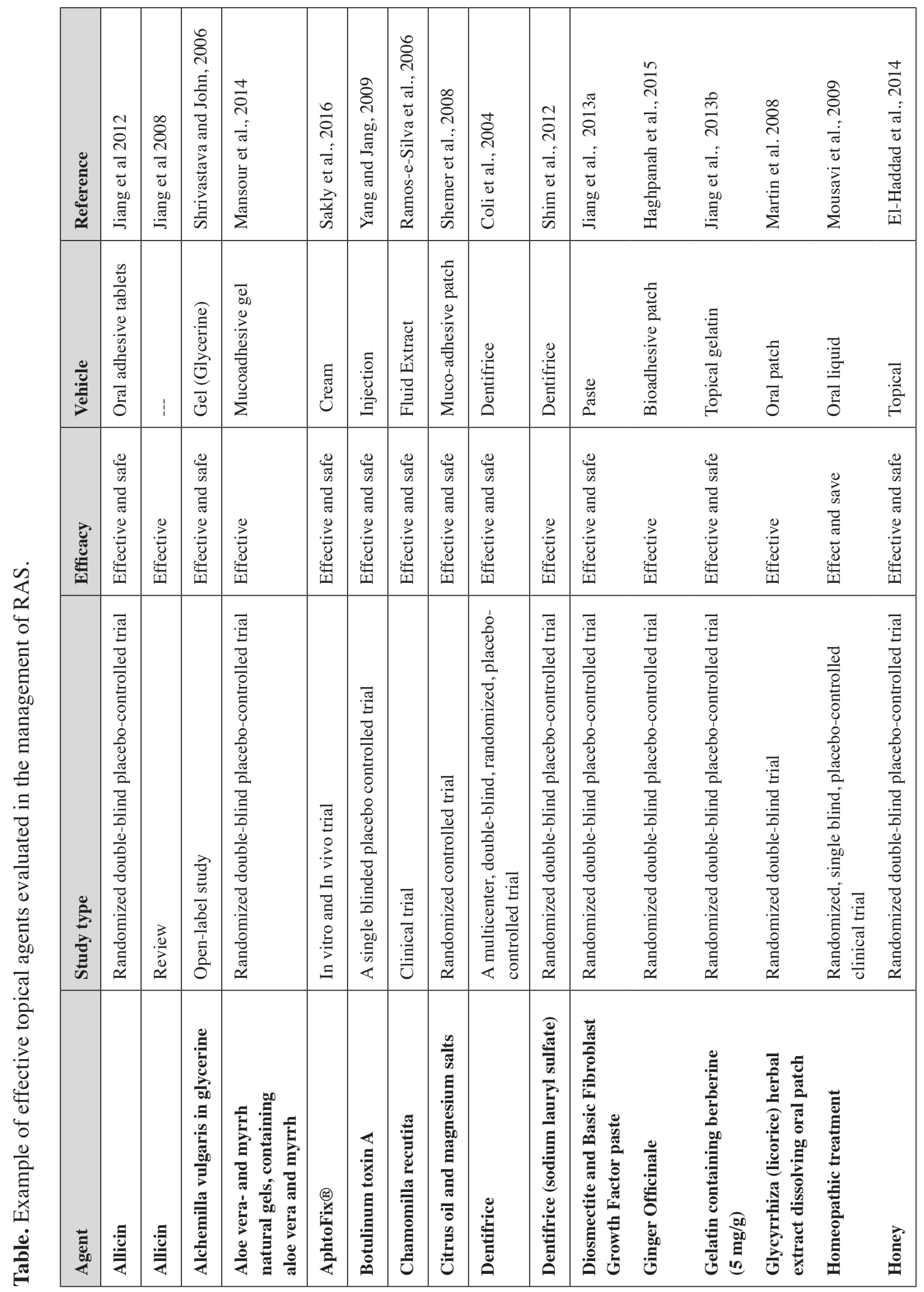




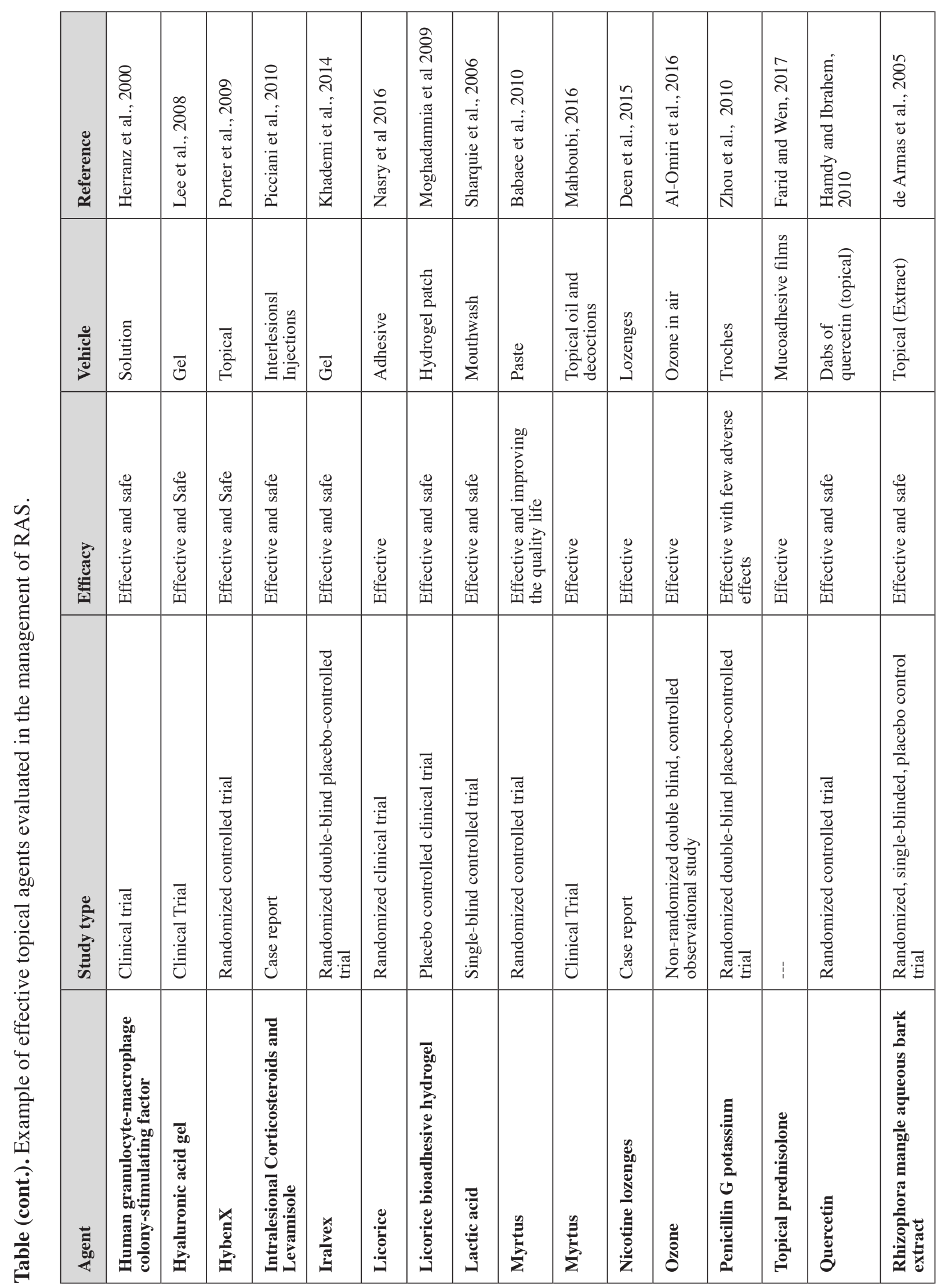




\section{Others}

Many other agents have also been used in the management of RAS. Few additional agents that are effective in the management of RAS have been summarized in the table below.

\section{CONCLUSION}

In conclusion, there is no single widely accepted treatment protocol for RAS. Most treatment modalities reduced pain and ulcer size. Topical corticosteroids are still considered to be the best mode of treatment of RAS, and they are effective in controlling symptomatic oral ulceration. These agents are easy to use with few adverse sides. Multicenter randomized controlled studies are needed to measure the real effect.

\section{REFERENCES}

1. Anand V, Gulati M, Govila V, Anand B (2013). Low-level laser therapy in the treatment of aphthous ulcer. Indian $\mathrm{J}$ Dent Res. 24:267-70.

2. Abbasi F, Raoof M, Khatami R, Shadman N, BorjianBoroojeni F, Nazari F (2016). Effectiveness of Amlexanox and Adcortyl for the treatment of recurrent aphthous ulcers. J Clin Exp Dent. 8: e368-e372.

3. Addy M, Hunter L (1987). The effects of a $0.2 \%$ chlorhexidine gluconate mouthrinse on plaque, toothstaining and candida in aphthous ulcer patients. A double-blind placebo-controlled cross-over study. J Clin Periodontol. 14:267-273.

4. Albrektson M, Hedström L, Bergh H (2014). Recurrent aphthous stomatitis and pain management with low-level laser therapy: a randomized controlled trial. Oral Surg Oral Med Oral Pathol Oral Radiol. 117:590-594.

5. Alidaee MR, Taheri A, Mansoori P, Ghodsi SZ (2005). Silver nitrate cautery in aphthous stomatitis: a randomized controlled trial. Br J Dermatol.153:521-525.

6. Al-Na'mah ZM, Carson R, Thanoon IA (2009). Dexamucobase: a novel treatment for oral aphthous ulceration. Quintessence Int. 40:399-404.

7. Al-Omiri MK, Alhijawi M, AlZarea BK, Abul Hassan RS, Lynch E (2016). Ozone treatment of recurrent aphthous stomatitis: a double blinded study. Sci Rep. 6:27772.
8. Altaei DT (2012). Topical lavender oil for the treatment of recurrent aphthous ulceration. Am J Dent. 25:39-43.

9. Babaee N, Mansourian A, Momen-Heravi F, Moghadamnia A, Momen-Beitollahi J (2010). The efficacy of a paste containing Myrtus communis (Myrtle) in the management of recurrent aphthous stomatitis: a randomized controlled trial. Clin Oral Investig. 14:65-70.

10. Bardellini E, Amadori F, Conti G, Majorana A (2016) Clinical efficacy of a solution composed by sodium bicarbonate and alginate, aloe vera, propoli, chamomile, calendula and honey, in the treatment of minor recurrent aphthous stomatitis in children. Minerva Pediatr. 68:507509.

11. Belenguer-Guallar I, Jiménez-Soriano Y, ClaramuntLozano A (2014). Treatment of recurrent aphthous stomatitis. A literature review. J Clin Exp Dent. 6:e168174.

12. Bell J (2005). Amlexanox for the treatment of recurrent aphthous ulcers. Clin Drug Investig. 25:555-566.

13. Bhat S, Sujatha D (2013). A clinical evaluation of 5\% amlexanox oral paste in the treatment of minor recurrent aphthous ulcers and comparison with the placebo paste: a randomized, vehicle controlled, parallel, single center clinical trial. Indian J Dent Res. 24:593-598.

14. Casale M, Moffa A, Vella P, Rinaldi V, Lopez MA Grimaldi V, Salvinelli F (2017). Systematic review: the efficacy of topical hyaluronic acid on oral ulcers. J Biol Regul Homeost Agents. 31:63-69.

15. Coli P, Jontell M, Hakeberg M (2004). The effect of a dentifrice in the prevention of recurrent aphthous stomatitis. Oral Health Prev Dent. 2:133-141.

16. Darshan DD, Kumar CN, Kumar AD, Manikantan NS, Balakrishnan D, Uthkal MP (2014). Clinical study to know the efficacy of Amlexanox 5\% with other topical Antiseptic, Analgesic and Anesthetic agents in treating minor RAS. J Int Oral Health. 6:5-11.

17. de Armas E, Sarracent Y, Marrero E, Fernández O, Branford-White C (2005). Efficacy of Rhizophora mangle aqueous bark extract (RMABE) in the treatment of aphthous ulcers: a pilot study. Curr Med Res Opin. 21:1711-1715.

18. Deen K, Curchin C, Wu J (2015). Successful treatment of recurrent aphthous ulcers with nicotine lozenges in a lifelong non-smoker. Australas J Dermatol. 56:143-144. 
19. Edres MA, Scully C, Gelbier M (1997). Use of proprietary agents to relieve recurrent aphthous stomatitis. Br Dent J.182:144-146.

20. El-Haddad SA, Asiri FY, Al-Qahtani HH, Al-Ghmlas AS (2014). Efficacy of honey in comparison to topical corticosteroid for treatment of recurrent minor aphthous ulceration: a randomized, blind, controlled, parallel, double-center clinical trial. Quintessence Int. 45:691-701.

21. Farid RM, Wen MM (2017). Promote Recurrent Aphthous Ulcer Healing with Low Dose Prednisolone Bilayer Mucoadhesive Buccal Film. Curr Drug Deliv. 14:123-135.

22. Gorsky M, Epstein J, Rabenstein S, Elishoov H, Yarom $N$ (2007). Topical minocycline and tetracycline rinses in treatment of recurrent aphthous stomatitis: a randomized cross-over study. Dermatol Online J.13:1.

23. Gorsky M, Epstein J, Raviv A, Yaniv R, Truelove E (2008) Topical minocycline for managing symptoms of recurrent aphthous stomatitis. Spec Care Dentist. 28:27-31.

24. Graykowski EA, Kingman A (1978). Double-blind trial of tetracycline in recurrent aphthous ulceration. J Oral Pathol. 7:376-382.

25. Greer RO Jr, Lindenmuth JE, Juarez T, Khandwala A (1993). A double-blind study of topically applied 5\% amlexanox in the treatment of aphthous ulcers. J Oral Maxillofac Surg. 51:243-248.

26. Haghpanah P, Moghadamnia AA, Zarghami A, Motallebnejad M (2015). Muco-bioadhesive containing ginger officinal e extract in the management of recurrent aphthous stomatitis: A randomized clinical study. Caspian J Intern Med. 6:3-8.

27. Hamdy AA, Ibrahem MA (2010). Management of aphthous ulceration with topical quercetin: a randomized clinical trial. J Contemp Dent Pract.11:E009-16.

28. Han M, Fang H, Li QL, Cao Y, Xia R, Zhang ZH (2016). Effectiveness of Laser Therapy in the Management of Recurrent Aphthous Stomatitis: A Systematic Review. Scientifica (Cairo). 2016;9062430.

29. Herranz P, Arribas JR, Navarro A, Peña JM, González J, Rubio FA, Casado M (2000). Successful treatment of aphthous ulcerations in AIDS patients using topical granulocyte-macrophage colony-stimulating factor. $\mathrm{Br} \mathrm{J}$ Dermatol. 142:171-176.

30. Holbrook WP, Kristmundsdóttir T, Loftsson T (1998). Aqueous hydrocortisone mouthwash solution: clinical evaluation. Acta Odontol Scand. 56:157-160.
31. Hoseinpour H, Peel SA, Rakhshandeh H, Forouzanfar A, Taheri M, Rajabi O, Saljoghinejad M, Sohrabi K (2011). Evaluation of Rosa damascena mouthwash in the treatment of recurrent aphthous stomatitis: a randomized, doubleblinded, placebo-controlled clinical trial. Quintessence Int. 42:483-91.

32. Jiang XW, Hu J, Mian FI (2008). A new therapeutic candidate for oral aphthous ulcer: Allicin. Med Hypotheses. 71:897-899.

33. Jiang XW, Zhang Y, Song GD, Li FF, Peng HY, Yang SK, Sun GL (2012). Clinical evaluation of allicin oral adhesive tablets in the treatment of recurrent aphthous ulceration. Oral Surg Oral Med Oral Pathol Oral Radiol. 113:500-504.

34. Jiang XW, Zhang Y, Zhang H, Lu K, Yang SK, Sun GL (2013a). Double-blind, randomized, controlled clinical trial of the effects of diosmectite and basic fibroblast growth factor paste on the treatment of minor recurrent aphthous stomatitis. Oral Surg Oral Med Oral Pathol Oral Radiol. 116:570-575.

35. Jiang XW, Zhang Y, Zhu YL, Zhang H, Lu K, Li FF, Peng HY (2013b). Effects of berberine gelatin on recurrent aphthous stomatitis: a randomized, placebo-controlled, double-blind trial in a Chinese cohort. Oral Surg Oral Med Oral Pathol Oral Radiol. 115:212-217.

36. Jijin MJ, Rakaraddi M, Pai J, Jaishankar HP, Krupashankar R, Kavitha AP, Anjana R, Shobha R (2016) Low-level laser therapy versus $5 \%$ amlexanox: a comparison of treatment effects in a cohort of patients with minor aphthous ulcers. Oral Surg Oral Med Oral Pathol Oral Radiol. 121:269-73.

37. Jurge S, Kuffer R, Scully C, Porter SR (2006). Mucosal disease series. Number VI. Recurrent aphthous stomatitis. Oral Dis.12:1-21.

38. Keenan AV (2012). Evid Based Dent.13:75.

39. Khademi H, Iranmanesh P, Moeini A, Tavangar A (2014). Evaluation of the Effectiveness of the Iralvex Gel on the Recurrent Aphthous Stomatitis Management. Int Sch Res Notices. 2014:175378.

40. Khandwala A, Van Inwegen RG, Alfano MC (1997). 5\% amlexanox oral paste, a new treatment for recurrent minor aphthous ulcers: I. Clinical demonstration of acceleration of healing and resolution of pain. Oral Surg Oral Med Oral Pathol Oral Radiol Endod. 83:222-230.

41. Lee JH, Jung JY, Bang D (2008). The efficacy of topical $0.2 \%$ hyaluronic acid gel on recurrent oral ulcers: 
comparison between recurrent aphthous ulcers and the oral ulcers of Behçet's disease. J Eur Acad Dermatol Venereol. 22:590-595.

42. Liu C, Zhou Z, Liu G, Wang Q, Chen J, Wang L, Zhou Y, Dong G, Xu X, Wang Y, Guo Y, Lin M, Wu L, Du G, Wei C, Zeng X, Wang X, Wu J, Li B, Zhou G, Zhou H (2012). Efficacy and safety of dexamethasone ointment on recurrent aphthous ulceration. Am J Med.125:292-301.

43. Liu J, Zeng X, Chen Q, Cai Y, Chen F, Wang Y, Zhou H, Lin M, Shi J, Wang Z, Zhang Y (2006). An evaluation on the efficacy and safety of amlexanox oral adhesive tablets in the treatment of recurrent minor aphthous ulceration in a Chinese cohort: a randomized, double-blind, vehiclecontrolled, unparallel multicenter clinical trial. Oral Surg Oral Med Oral Pathol Oral Radiol Endod. 102:475-81.

44. Lo Muzio L, della Valle A, Mignogna MD, Pannone G, Bucci P, Bucci E, Sciubba J (2001). The treatment of oral aphthous ulceration or erosive lichen planus with topical clobetasol propionate in three preparations: a clinical and pilot study on 54 patients. J Oral Pathol Med. 30:611-617.

45. Lozada-Nur F, Huang MZ, Zhou GA (1991). Open preliminary clinical trial of clobetasol propionate ointment in adhesive paste for treatment of chronic oral vesiculoerosive diseases. Oral Surg Oral Med Oral Pathol. 71:283-287.

46. Mahboubi M. (2016). Myrtus communis L. and its application in treatment of Recurrent Aphthous Stomatitis. J Ethnopharmacol. 193:481-489.

47. Mansour G, Ouda S, Shaker A, Abdallah HM (2014). Clinical efficacy of new aloe vera- and myrrh-based oral mucoadhesive gels in the management of minor recurrent aphthous stomatitis: a randomized, double-blind, vehiclecontrolled study. J Oral Pathol Med. 43:405-9.

48. Martin MD, Sherman J, van der Ven P, Burgess J (2008). A controlled trial of a dissolving oral patch concerning glycyrrhiza (licorice) herbal extract for the treatment of aphthous ulcers. Gen Dent. 56:206-210.

49. Meng W, Dong Y, Liu J, Wang Z, Zhong X, Chen R, Zhou H, Lin M, Jiang L, Gao F, Xu T, Chen Q, Zeng X (2009). A clinical evaluation of amlexanox oral adhesive pellicles in the treatment of recurrent aphthous stomatitis and omparison with amlexanox oral tablets: a randomized, placebo controlled, blinded, multicenter clinical trial. Trials. 10:30.
50. Miles DA, Bricker SL, Razmus TF, Potter RH (1993). Triamcinolone acetonide versus chlorhexidine for treatment of recurrent stomatitis. Oral Surg Oral Med Oral Pathol.75:397-402.

51. Misra N, Maiti D, Misra P, Singh AK (2013). 940 nm diode laser therapy in management of recurrent apthous ulcer. BMJ Case Rep. 2013.

52. Moghadamnia AA, Motallebnejad M, Khanian M (2009). The efficacy of the bioadhesive patches containing licorice extract in the management of recurrent aphthous stomatitis Phytother Res. 23:246-250.

53. Mousavi F, Mojaver YN, Asadzadeh M, Mirzazadeh M (2009). Homeopathic treatment of minor aphthous ulcer: a randomized, placebo-controlled clinical trial. Homeopathy. 98:137-141.

54. Murray B, Biagioni PA, Lamey PJ (2006). The efficacy of amlexanox Ora Disc on the prevention of recurrent minor aphthous ulceration. J Oral Pathol Med. 35:117-122.

55. Murray B, McGuinness N, Biagioni P, Hyland P, Lamey PJ (2005). A comparative study of the efficacy of Aphtheal in the management of recurrent minor Aphthous ulceration. J Oral Pathol Med. 34:413-419.

56. Najeeb S, Khurshid Z, Zohaib S, Najeeb B, Qasim SB, Zafar MS (2016). Management of recurrent aphthous ulcers using low-level lasers: A systematic review. Medicina (Kaunas). 52:263-268.

57. Nasry SA, El Shenawy HM, Mostafa D, Ammar NM (2016). Different modalities for treatment of recurrent aphthous stomatitis. A Randomized clinical trial. J Clin Exp Dent. 1; 8:e517-e522.

58. Nolan A, Baillie C, Badminton J, Rudralingham M, Seymour RA (2006). The efficacy of topical hyaluronic acid in the management of recurrent aphthous ulceration. Oral Pathol Med. 35:461-465.

59. Ofluoglu D, Ergun S, Warnakulasuriya S, Namdar-Pekiner F, Tanyeri H (2017). An evaluation of the efficacy of a topical gel with Triester Glycerol Oxide (TGO) in the treatment of minor recurrent aphthous stomatitis in a Turkish cohort: A randomized, double-blind, placebocontrolled clinical trial. Med Oral Patol Oral Cir Bucal. 22:e159-e166.

60. Pentapati KC, Smriti K, Gadicherla S (2016). Low-level laser therapy versus 5\% amlexanox: a comparison of treatment effects in a cohort of minor aphthous ulcers 
patients-a commentary. Oral Surg Oral Med Oral Pathol Oral Radiol.122:373.

61. Picciani BL, Silva-Junior GO, Barbirato DS, Ramos RT, Cantisano MH (2010). Regression of major recurrent aphthous ulcerations using a combination of intralesional corticosteroids and levamisole: a case report. Clinics (Sao Paulo). 65:650-652.

62. Pimlott SJ, Walker DM (1983). A controlled clinical trial of the efficacy of topically applied fluocinonide in the treatment of recurrent aphthous ulceration. Br Dent J. 154:174-177.

63. Porter SR, Al-Johani K, Fedele S, Moles DR (2009). Randomised controlled trial of the efficacy of HybenX in the symptomatic treatment of recurrent aphthous stomatitis. Oral Dis.15:155-61.

64. Prasad RS, Pai A (2013). Assessment of immediate pain relief with laser treatment in recurrent aphthous stomatitis. Oral Surg Oral Med Oral Pathol Oral Radiol.116:189-193.

65. Quijano D, Rodríguez M (2008). [Topical corticosteroids in recurrent aphthous stomatitis. Systematic review]. Acta Otorrinolaringol Esp. 59:298-307.

66. Ramos-e-Silva M, Ferreira AF, Bibas R, Carneiro S (2006). Clinical evaluation of fluidextract of Chamomilla recutita for oral aphthae. J Drugs Dermatol. 5:612-7.

67. Rhodus NL, Bereuter J (1998). An evaluation of a chemical cautery agent and an anti-inflammatory ointment for the treatment of recurrent aphthous stomatitis: a pilot study. Quintessence Int. 29:769-773.

68. Rodríguez M, Rubio JA, Sanchez R (2007). Effectiveness of two oral pastes for the treatment of recurrent aphthous stomatitis. Oral Dis.13:490-504.

69. Rodríguez-Archilla A, Raissouni T (2017). Randomized clinical trial of the effectiveness of complementary therapies for recurrent aphthous stomatitis. Med Clin (Barc).149:55-60.

70. Sakly A, De Wever B, Jutla B, Satia M, Bogaert JP (2016). The safety and efficacy of AphtoFix ${ }^{\circledR}$ mouth ulcer cream in the management of recurrent aphthous stomatitis. BMC Oral Health.16:17.

71. Scully C, Gorsky M, Lozada-Nur F (2003). The diagnosis and management of recurrent aphthous stomatitis: a consensus approach. J Am Dent Assoc. 134:200-207.
72. Shao Y, Zhou H (2016). Clinical evaluation of a toothpaste containing lysozyme for the treatment of recurrent aphthous stomatitis: A 3-month, double-blind, randomized study. Am J Dent. 29:303-306.

73. Sharquie KE, Al-Tammimy SM, Al-Mashhadani S, Hayani RK, Al-Nuaimy AA (2006). Lactic acid 5 percent mouthwash is an effective mode of therapy in treatment of recurrent aphthous ulcerations. Dermatol Online J. 12:2.

74. Shemer A, Amichai B, Trau H, Nathansohn N, Mizrahi B, Domb AJ (2008). Efficacy of a mucoadhesive patch compared with an oral solution for treatment of aphthous stomatitis. Drugs R D. 9:29-35.

75. Shim YJ, Choi JH, Ahn HJ, Kwon JS (2012). Effect of sodium lauryl sulfate on recurrent aphthous stomatitis: a randomized controlled clinical trial. Oral Dis. 18:655-60.

76. Shrivastava R, John GW (2006). Treatment of Aphthous Stomatitis with topical Alchemilla vulgaris in glycerine. Clin Drug Investig. 26:567-73.

77. Skaare AB, Herlofson BB, Barkvoll P (1996). Mouthrinses containing triclosan reduce the incidence of recurrent aphthous ulcers (RAU). J Clin Periodontol. 23:778-781.

78. Skulason S, Holbrook WP, Kristmundsdottir T (2009). Clinical assessment of the effect of a matrix metalloproteinase inhibitor on aphthous ulcers. Acta Odontol Scand. 67:25-29.

79. Soylu Özler G (2014). Silver nitrate cauterization: a treatment option for aphthous stomatitis. J Craniomaxillofac Surg. 42:e281-283.

80. Sun A, Chia JS, Wang WB, Chiang CP (2005). "TienHsien liquid" can modulate antigen-stimulated cytokine production by T-cells isolated from patients with recurrent aphthous ulcerations. Am J Chin Med. 33:559-571.

81. Suter VGA, Sjölund S, Bornstein MM (2017). Effect of laser on pain relief and wound healing of recurrent aphthous stomatitis: a systematic review. Lasers Med Sci. 32:953-963.

82. Teixeira F, Mosqueda-Taylor A, Montaño S, DomínguezSoto L (1999). Treatment of recurrent oral ulcers with mometasone furoate lotion. Postgrad Med J. 75:574.

83. Tezel A, Kara C, Balkaya V, Orbak R (2009). An evaluation of different treatments for recurrent aphthous stomatitis and patient perceptions: Nd:YAG laser versus medication. Photomed Laser Surg. 27:101-106. 
84. Vidal A, Sabatini M, Rolland-Valognes G, Renard P, Madelmont JC, Mounetou E (2007). Synthesis and in vitro evaluation of targeted tetracycline derivatives: effects on inhibition of matrix metalloproteinases. Bioorg Med Chem. 15:2368-2374.

85. Vijayabala GS, Kalappanavar AN, Annigeri RG, Sudarshan R, Shettar SS (2013). Single application of topical doxycycline hyclate in the management of recurrent aphthous stomatitis. Oral Surg Oral Med Oral Pathol Oral Radiol. 116:440-446.

86. Vincent SD, Lilly GE (1992). Clinical, historic, and therapeutic features of Aphthous stomatitis. Literature review and open clinical trial employing steroids. Oral Surg Oral Med Oral Pathol. 74:79-86.

87. Yang TY, Jang TY (2009). The value of local botulinum toxin A injection in the treatment of the pain of aphthous ulcer. Eur Arch Otorhinolaryngol. 266:445-8.

88. Yarom N, Zelig K, Epstein JB, Gorsky M (2017). The efficacy of minocycline mouth rinses on the symptoms associated with recurrent aphthous stomatitis: a randomized, double-blind, crossover study assessing different doses of oral rinse. Oral Surg Oral Med Oral Pathol Oral Radiol. 123:675-679.

89. Yel L, Tezcan I, Hasturk H, Ersoy F, Sanal O, Yavuzyilmaz E (1994). Oral findings, treatment and follow-up of a case with major aphthous stomatitis (Sutton's disease). J Clin Pediatr Dent.19:49-53.

90. Yilmaz HG, Albaba MR, Caygur A, Cengiz E, BokeKaracaoglu F, Tumer H (2017). Treatment of recurrent aphthous stomatitis with Er,Cr:YSGG laser irradiation: A randomized controlled split mouth clinical study. J Photochem Photobiol B.170:1-5.

91. Zand N, Fateh M, Ataie-Fashtami L, Djavid GE, Fatemi SM, Shirkavand A (2012). Promoting wound healing in minor recurrent aphthous stomatitis by non-thermal, nonablative $\mathrm{CO}(2)$ laser therapy: a pilot study. Photomed Laser Surg. 30:719-723.

92. Zeini Jahromi N, Ghapanchi J, Pourshahidi S, Zahed M, Ebrahimi H (2017). Clinical Evaluation of High and LowLevel Laser Treatment (CO2vsInGaAlP Diode Laser) for Recurrent Aphthous Stomatitis. J Dent (Shiraz). 18:17-23.

93. Zhou Y, Chen Q, Meng W, Jiang L, Wang Z, Liu J, Lin M, Zhou H, Chen X, Zhao M,Zeng X (2010). Evaluation of penicillin $G$ potassium troches in the treatment of minor recurrent aphthous ulceration in a Chinese cohort: a randomized, double-blinded, placebo and no-treatmentcontrolled, multicenter clinical trial. Oral Surg Oral Med Oral Pathol Oral Radiol Endod.109:561-566. 